\title{
Within-Hemifield Competition in Early Visual Areas Limits the Ability to Track Multiple Objects with Attention
}

\author{
Viola S. Störmer, ${ }^{1}$ George A. Alvarez, ${ }^{1}$ and Patrick Cavanagh ${ }^{1,2}$ \\ ${ }^{1}$ Department of Psychology, Harvard University, Cambridge, Massachusetts 02138, and ${ }^{2}$ Laboratoire Psychologie de la Perception, Université Paris \\ Descartes, Sorbonne Paris Cité, CNRS UMR 8242, Paris, France
}

It is much easier to divide attention across the left and right visual hemifields than within the same visual hemifield. Here we investigate whether this benefit of dividing attention across separate visual fields is evident at early cortical processing stages. We measured the steady-state visual evoked potential, an oscillatory response of the visual cortex elicited by flickering stimuli, of moving targets and distractors while human observers performed a tracking task. The amplitude of responses at the target frequencies was larger than that of the distractor frequencies when participants tracked two targets in separate hemifields, indicating that attention can modulate early visual processing when it is divided across hemifields. However, these attentional modulations disappeared when both targets were tracked within the same hemifield. These effects were not due to differences in task performance, because accuracy was matched across the tracking conditions by adjusting target speed (with control conditions ruling out effects due to speed alone). To investigate later processing stages, we examined the $\mathrm{P} 3$ component over central-parietal scalp sites that was elicited by the test probe at the end of the trial. The P3 amplitude was larger for probes on targets than on distractors, regardless of whether attention was divided across or within a hemifield, indicating that these higher-level processes were not constrained by visual hemifield. These results suggest that modulating early processing stages enables more efficient target tracking, and that within-hemifield competition limits the ability to modulate multiple target representations within the hemifield maps of the early visual cortex.

Key words: EEG/SSVEP; hemifield independence; tracking; visual attention

\section{Introduction}

By directing attention to a specific region in a visual scene, information at the attended location can be selected for enhanced processing (LaBerge, 1995). Traditionally, attention is thought of as a centrally organized process that controls selection similarly along the entire processing stream (Broadbent, 1958; Eriksen and Yeh, 1985; Moran and Desimone, 1985). Recent studies, however, suggest that attentional mechanisms are fundamentally constrained by properties of lower-level cortical areas. For example, it is easier to process multiple targets across the left and right visual hemifields, than within the same visual hemifield (Sereno and Kosslyn, 1991; Awh and Pashler, 2000; Chakravarthi and Cavanagh, 2009; Reardon et al., 2009; Alvarez et al., 2012). These findings suggest that separate attentional resources exist for the left and right visual fields to select multiple targets, effectively doubling the resources available when targets are distributed in

\footnotetext{
Received March 12, 2014; revised May 26, 2014; accepted June 30, 2014.

Author contributions: V.S.S., G.A.A., and P.C. designed research; V.S.S. performed research; V.S.S. analyzed data; V.S.S., G.A.A., and P.C. wrote the paper.

This work was supported by a Marie Curie fellowship (European Union Grant PIOF-GA-2012-329920 to V.S.S.), a National Science Foundation Faculty Early Career Development Program award (BCS-0953730 to G.A.A.), and a National Institutes of Health grant (EY09258) and a Chaire d'Excellence and European Research Council Position grant (to P.C.). We thank Phillip J. Holcombe at Tufts University for his generosity in providing space and time in his EEG laboratory for pilot experiments on this project.

The authors declare no competing financial interests.

Correspondence should be addressed to Viola Störmer at the above address. E-mail: vstormer@fas.harvard.edu. DOI:10.1523/JNEUROSCI.0980-14.2014

Copyright $\odot 2014$ the authors $\quad 0270-6474 / 14 / 3311526-08 \$ 15.00 / 0$
}

both visual fields compared with when they are all in only one visual hemifield.

This across-hemifield advantage is particularly pronounced in tasks that require sustained selection of targets from distractors, such as attentional tracking: participants can track twice as many target objects among distractors when they appear in separate visual fields compared with when they appear in the same hemifield (Alvarez and Cavanagh, 2005; Holcombe and Chen, 2012; Hudson et al., 2012). These findings are consistent with the possibility that hemifield independence arises due to limitations at the earliest stages of visual processing - during sensory processing. If this were the case, sensory enhancement in visual areas should be larger for targets presented across hemifields relative to targets that are presented within the same hemifield. The present study used electrophysiological recordings to measure processing at early stages directly, enabling us to assess whether hemifield independence is evident during early sensory processing.

Specifically, the present study combines behavioral measures and electrophysiological recordings of steady-state visual evoked potentials (SSVEPs) over visual cortex and event-related potentials (ERPs) in an attentive tracking task (for a similar approach using a detection task, see Walter et al., 2014). Participants tracked one target or tracked two targets that either appeared in separate visual fields (bottom-left and bottom-right) or in the same visual hemifield (bottom-right and top-right). Performance was matched across tracking conditions by adjusting target speed. Early visual processing of targets and distractors was 
A

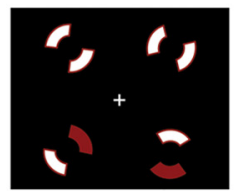

$800 \mathrm{~ms}$

\section{Trial sequence}

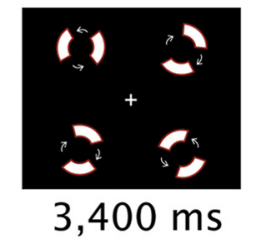

\section{Response \#1 Response \#2}

B

\section{Rotation speed limits}

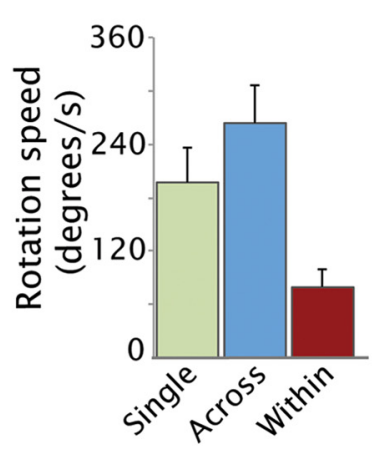

\section{C}
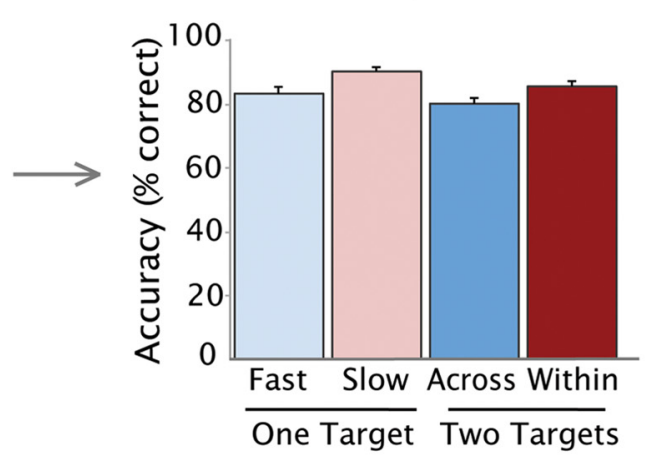

Figure 1. Experimental procedure and behavioral results. $\boldsymbol{A}$, Illustration of an example trial in which participants tracked two targets across hemifields (bottom-left and bottom-right quadrant). Targets were highlighted in red at the beginning of the trial, and then turned back to look identical to the distractors. During the rotation period, each target and distractor flickered at a separate frequency (see Materials and Methods for details). $\boldsymbol{B}$, Speed thresholds that were obtained before the EEG task show clear hemifield effects, such that threshold rotation speeds were much faster when participants tracked two targets in separate visual hemifields (across) relative to two targets in the same visual hemifield (within), but not different from tracking a single target. $\boldsymbol{C}$, After adjusting individual speed thresholds, accuracy rates during the EEG session show no significant differences between acrosshemifield versus within-hemifield tracking. Error bars represent the SEM.

quantified by measuring the SSVEPs elicited by these stimuli (Störmer et al., 2013a). To assess later processing stages related to identification and discrimination processes, the P3 component was examined (Polich, 2007).

We hypothesized that there would be greater modulation of SSVEP responses in early visual areas when attention was divided across hemifields than within a hemifield. This would provide direct evidence that attention cannot modulate early visual responses for multiple items within a hemifield, and would suggest that this constraint underlies the across-hemifield advantage. Furthermore, if later higher-order processes indexed by the P3 component show no effects of hemifield, this would suggest that attentional processing limits consist of a set of distinct constraints along the processing chain, and that their interplay, rather than one unified boundary, ultimately determines attentional capacity limits.

\section{Materials and Methods \\ Participants}

Fifteen volunteers participated in the experiment after giving informed consent. Data from two participants had to be excluded from the analysis because $>40 \%$ of their trials were rejected as a result of artifacts in the EEG; data from one other participant were excluded because of technical problems with the eye tracker during the experiment. Of the remaining 12 participants (age range, 18-28 years; eight female), all were righthanded and reported normal or corrected-to-normal vision.

Stimuli

The stimuli were white arcs (quarter of a circle; $2.5^{\circ}$ inner boundary, $1.5^{\circ}$ width; luminance, $132 \mathrm{~cd} / \mathrm{m}^{2}$ ) with a narrow red outline (Fig. $1 A$ ). A pair

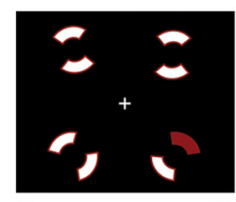

of arc stimuli was presented in each quadrant of the visual field, $7^{\circ}$ from the center of the screen. The diameter of the inner circle of the two arcs measured $2.8^{\circ}$. On each trial, the direction of rotation (clockwise or counterclockwise) was determined randomly and independently for each arc pair, and throughout the tracking period the rotation direction changed at random intervals and independently of each other, moving in the same direction for $\geq 500 \mathrm{~ms}$, but never $>3000 \mathrm{~ms}$. Within each arc pair, the motion direction change was not simultaneous for the two items, such that the arcs in a pair moved asynchronous of each other, but never touched each other. This prevented observers from tracking the two arcs as a single perceptual unit.

Each arc stimulus flickered at a distinct frequency during the rotation period, allowing SSVEP responses to be aligned with particular items in the display. In the upper-right quadrant, one of the arcs flickered at $\sim 7.7 \mathrm{~Hz}$ (mean luminance, $60.2 \mathrm{~cd} / \mathrm{m}^{2}$ ), and the other one at $\sim 9.4 \mathrm{~Hz}$ (mean luminance, $58.8 \mathrm{~cd} / \mathrm{m}^{2}$ ); in the lower-right quadrant, one of the arcs flickered at $\sim 10.6 \mathrm{~Hz}$ (mean luminance, $66.2 \mathrm{~cd} / \mathrm{m}^{2}$ ), and the other one at $\sim 12.2 \mathrm{~Hz}$ (mean luminance, $56.7 \mathrm{~cd} / \mathrm{m}^{2}$ ); in the lower-left quadrant, one of the arcs flickered at $\sim 14.2 \mathrm{~Hz}$ (mean luminance, $\left.66.0 \mathrm{~cd} / \mathrm{m}^{2}\right)$, and the other one at $\sim 17.0 \mathrm{~Hz}$ (mean luminance, $53.0 \mathrm{~cd} / \mathrm{m}^{2}$ ); last, in the upper-left quadrant, one of the arcs flickered at $\sim 21.3 \mathrm{~Hz}$ (mean luminance, $66.1 \mathrm{~cd}$ / $\mathrm{m}^{2}$ ), and the other one at $\sim 28.3 \mathrm{~Hz}$ (mean luminance, $44.1 \mathrm{~cd} / \mathrm{m}^{2}$ ). These distinct frequencies allowed the clear separation of target and distractor processing within each stimulus pair in the SSVEP responses. The SSVEP is the oscillatory potential field that is generated by the visual cortex in response to flickering stimuli that has the same fundamental frequency as the driving stimulus (Regan, 1989). Its amplitude is increased for attended compared with unattended stimuli (Morgan et al., 1996; for review, see Andersen et al., 2011a). On half of the trials, the targets flickered at the lower frequency within a pair (i.e., 7.7, 10.6, 14.2 Hz), and the distractors flickered at the higher frequency (i.e., 9.4, 12.2, $17.0 \mathrm{~Hz}$ ); this assignment was reversed on the remaining half of the trials. To increase the number of trials and statistical power for targets in the upper-right, lower-left, and lower-right quadrants, the arcs in the upper-left quadrant were never assigned to be targets.

The test probe at the end of the trial consisted of a red flash $\left(6.9 \mathrm{~cd} / \mathrm{m}^{2}\right)$ that appeared on the target on half of the trials and on the distractor in the remaining half of the trials. The red test probe remained on the screen until the participant hit a response button indicating whether the probed item was a target. The ERP elicited by the red test probe enabled us to examine the P3 component over central-parietal scalp sites separately for probes on the target and the distractor (see Electrophysiological recordings and Analysis). This central-parietal P3 component reflects later identification and decision processes and is sensitive to the accuracy and confidence with which targets are discriminated from distractors (Squires et al., 1973; Hillyard and Picton, 1987; Polich, 2007).

\section{Procedure}

The experiment was conducted in a sound-attenuated and electrically shielded chamber that was dimly lit throughout the experiment. Participants were seated $57 \mathrm{~cm}$ in front of a 13 inch CRT monitor $(1024 \times 768$ pixels; refresh rate, $85 \mathrm{~Hz}$ ). The background color of the screen was black $\left(0.34 \mathrm{~cd} / \mathrm{m}^{2}\right)$ throughout the experiment. Participants were instructed to maintain their gaze at a white fixation cross $\left(0.6 \times 0.6^{\circ}\right)$ in the center 
of the screen throughout each trial. Fixation was controlled by tracking participants' eyes with a SMI RED-m eye tracker (SensoriMotor Instruments). When participants moved their gaze away from fixation $\left(>1.5^{\circ}\right)$ during the tracking period, the current trial was immediately aborted.

At the beginning of each trial, a target item within a stimulus pair was highlighted in red for $800 \mathrm{~ms}$. Then all stimuli turned back to white, flickering continuously at their respective frequencies from black to white (square function) and rotated for $3400 \mathrm{~ms}$. At the end of the trial, one of the items within a pair was highlighted in red and the participant had to indicate whether the probed item was a target or not by pressing the left or right button on a keyboard. When two targets were tracked, participants were asked about both items in a random order (Fig. $1 A$ ) and both test probes had to be correctly identified to count as a correct trial response. On half of the trials, the target stimulus within a pair was probed; on the remaining half of the trials, the distractor stimulus within a pair was probed.

Before the EEG experiment, rotation speed thresholds for $80 \%$ accuracy were obtained for each individual and condition: one target, two targets that were presented across visual hemifields, or two targets that were presented within one visual hemifield. Trial types were randomly intermixed and speed thresholds were computed online using Quest (Watson and Pelli, 1983). Participants performed 96 trials overall. The resulting speed thresholds were used in the EEG experiment to remove any performance differences between across-hemifield and withinhemifield tracking when there were two targets. Thus, participants tracked two targets that were presented in one of the following ways: (1) across hemifields (i.e., bottom-left and bottom-right) at a fast speed, (2) within a hemifield (i.e., bottom-right and top-right) at a slower speed so that performance was matched to the across-hemifields condition; or they tracked (3) one target at the same rotation speed ("fast") as during across-hemifield tracking (bottom-right), or (4) one target at the same rotation speed ("slow") as during within-hemifield tracking (bottomright). Testing a single target at both the fast and slow speed enabled us to determine whether speed differences alone would affect target and distractor processing. All trial types were randomly intermixed within each block. The EEG experiment consisted of 20 blocks of 32 trials each.

\section{Data analysis}

Behavioral analysis. Individual speed thresholds (degrees per second) were analyzed using repeated-measures ANOVA with tracking condition as the factor (single target, two targets across hemifields, two targets within a hemifield). To examine performance in the main EEG task, accuracy rates (percentage correct) were computed separately for the four tracking conditions: (1) single target at fast speed, (2) single target at slow speed, (3) two targets across hemifields (fast speed), (4) two targets within a hemifield (slow speed); and analyzed by means of a repeatedmeasures ANOVA. Follow-up pairwise comparisons were conducted to see at which level the effects were present.

Electrophysiological recordings and analysis. EEG was recorded continuously from $32 \mathrm{Ag} / \mathrm{AgCI}$ electrodes mounted in an elastic cap and amplified by an ActiCHamp amplifier (BrainVision). Electrodes were arranged according to the 10-10 system with three additional electrodes positioned inferior to the occipital sites to ensure adequate spatial sampling from the posterior scalp. The horizontal electro-oculogram was acquired using a bipolar pair of electrodes positioned at the external ocular canthi, and the vertical electro-oculogram was measured at electrode FP1, located above the left eye. All scalp electrodes were referenced to an electrode on the left mastoid, and were digitized at $500 \mathrm{~Hz}$. Continuous EEG data were filtered with a bandpass of $0.01-112.5 \mathrm{~Hz}$ offline. The EEG analysis was performed for correct trials only. Individual trials were rejected when contaminated with ocular artifacts. Blinks were detected using a semiautomated procedure, in which differences between minimum and maximum voltages were compared with a threshold value. Threshold values were determined by visual inspection for each subject individually (Green et al, 2008; cf. Störmer et al., 2013b). Eye movements were detected with the eye tracker during the recording (see
Procedure), and trials in which an eye movement occurred were excluded from the analysis. Artifact-free data were digitally rereferenced to the average reference.

The SSVEP was computed by a Fourier transform of the time interval 400-3380 ms after tracking onset. SSVEP amplitudes were quantified as the absolute value of the complex Fourier coefficients for each frequency; that is, for each participant and condition, the maximum absolute value within a small band of the respective stimulation frequency $( \pm 0.4 \mathrm{~Hz})$ was chosen, and then averaged across six posterior electrodes $(\mathrm{O} 1, \mathrm{Oz}$, $\left.\mathrm{O}_{2}, \mathrm{I} 3, \mathrm{Iz}, \mathrm{I} 4\right)$. The SSVEP amplitudes were normalized for each condition and frequency by dividing the amplitude by the mean amplitude of target and distractor for that particular frequency separately for each participant (Andersen et al., 2011b). The normalized amplitudes were then collapsed across frequencies to reveal amplitude values for targets and distractors in each of the conditions.

Our primary question is whether the magnitude of attentional modulation depends on tracking condition. Thus, we computed an index of attentional modulation by subtracting the normalized amplitude for distractors from the normalized amplitude for targets separately for each condition. The magnitude of attentional modulation across the different tracking conditions was then examined in two ways. First, to investigate how the attention effects would differ for within-hemifield versus acrosshemifield tracking, the indices of attentional modulation were collapsed across target location (i.e., across left and right targets in the acrosshemifield condition, and across top and bottom targets in the withinhemifield condition). These attentional modulations were computed separately for each subject and then analyzed with a repeated-measures ANOVA to test for effects of tracking condition.

Second, we compared attentional modulations separately for each target-distractor pair to rule out any effects due to speed alone. For example, we compared attentional modulation for one-target fast with attentional modulation at each of the target locations in the acrosshemifield condition (bottom-left and bottom-right quadrants), in which two targets moved at a fast speed. This additional analysis allowed us to directly compare the magnitude of attentional modulation for one target versus two targets while sensory input (i.e., flicker frequencies, target location, target speed) was matched (i.e., for targets in the bottom-right quadrant).

To examine later stages of target and distractor processing, we analyzed the P3 component of the averaged ERP to test probes presented at the end of each trial, either on a target or a distractor, for each tracking condition. The EEG was segmented into 1 s epochs starting $200 \mathrm{~ms}$ before the onset of the probe and the averaged ERP waveforms were digitally low-pass filtered $(-3 \mathrm{~dB}$ point at $25 \mathrm{~Hz})$. For each participant, the mean amplitude of the ERP waveforms elicited by the test probe was measured between 300 and $400 \mathrm{~ms}$ at three central-parietal electrode sites $(\mathrm{Cz}, \mathrm{CP} 1$, $\mathrm{CP} 2$ ). The resulting mean amplitudes were analyzed in a repeatedmeasures ANOVA with factors for test probe type (target vs distractor) and tracking condition (single fast, single slow, across hemifields, within a hemifield).

\section{Results}

\section{Behavior}

As expected, individual rotation speed thresholds for $80 \%$ accuracy differed between the single, across-hemifield, and withinhemifield conditions $\left(F_{(2,22)}=4.64, p=0.038, \eta^{2}=0.30\right)$. As shown in Figure $1 B$, speed limits for within-hemifield tracking were lower than both across-hemifield tracking $\left(t_{(11)}=3.49, p=\right.$ $\left.0.005, \eta^{2}=0.53\right)$ and one-target tracking $\left(t_{(11)}=2.51, p=0.029\right.$, $\left.\eta^{2}=0.36\right)$, but there was no significant difference between onetarget and across-hemifield tracking $\left(p=0.418, \eta^{2}=0.06\right)$. Thus, as shown in previous tracking tasks (Alvarez and Cavanagh, 2005; Holcombe and Chen, 2012), two targets could be tracked faster when they were presented across hemifields relative to when they were presented within a hemifield, but no slower than when they were presented as a single target. 
A

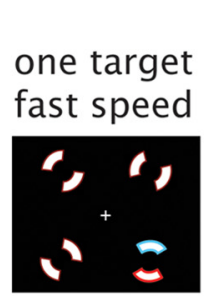

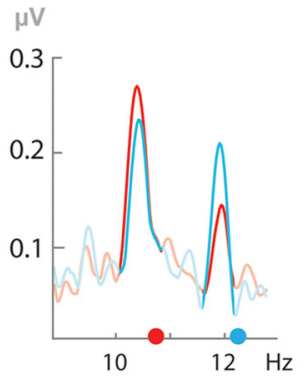

- Target at $10.7 \mathrm{~Hz}$ Target at $12.2 \mathrm{~Hz}$
B

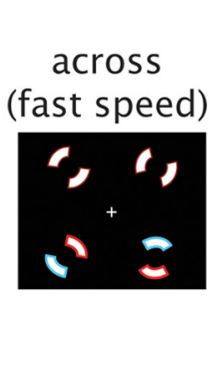

D

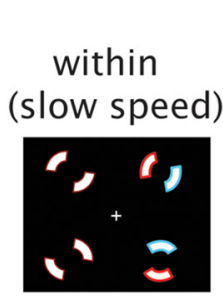

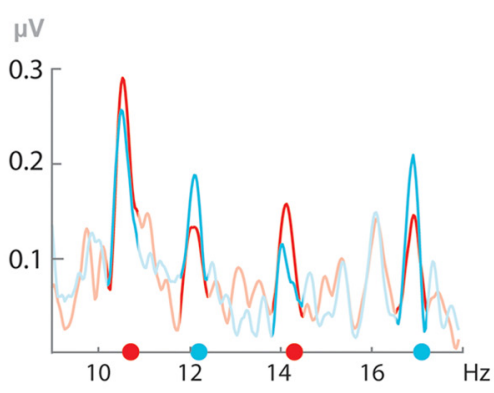

- Target at $10.7 \mathrm{~Hz}$ \& Target at $14.3 \mathrm{~Hz}$

- Target at $12.2 \mathrm{~Hz} \&$ Target at $17.0 \mathrm{~Hz}$
C

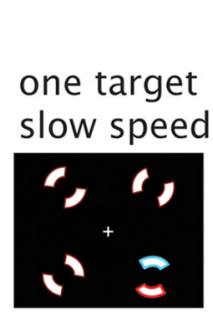

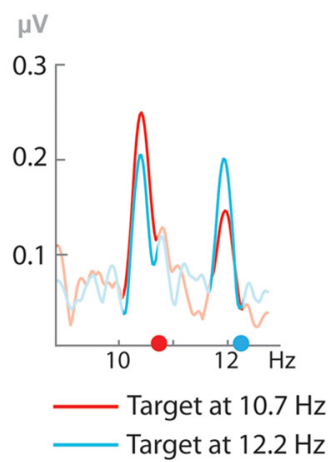

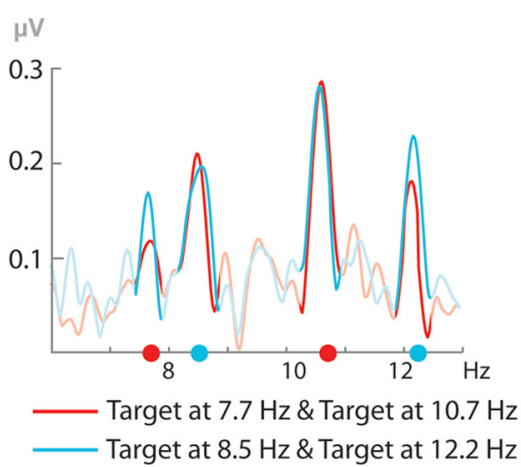

Figure 2. Grand-average amplitude spectrum across all subjects obtained by Fourier analysis of the SSVEP waveforms for targets and distractors across different frequencies, at occipital electrode Oz. Clear peaks are visible for the different stimulation frequencies across the different conditions. $A$, During single-target tracking (fast speed), the target elicits a larger SSVEP amplitude than the distractor. B, SSVEP amplitudes are larger for both targets relative to distractors during across-hemifield tracking (at fast speed). C, During single-target tracking (slow speed), the target elicits a larger SSVEP amplitude than the distractor. D, SSVEP amplitudes show less clear attentional modulations for targets and distractors during within-hemifield tracking.

To ensure that the observed effects were not due to differences in target location, we ran an additional group of subjects $(N=6)$ using the same tracking task and assessed individual speed thresholds separately for tracking two targets in the upper (i.e., top-left and top-right) and lower (i.e., bottom-left and bottomright) visual field, as well as for tracking two targets in the left (i.e., top-left and bottom-left) and right (i.e., top-right and bottomright) visual field. No reliable differences in speed thresholds were observed for upper $\left(261^{\circ} / \mathrm{s}\right.$; SEM, 31) versus lower $\left(277^{\circ} / \mathrm{s}\right.$; SEM, 27) visual field ( $p=0.63)$, or left $(128 \%$ s; SEM, 25) versus right $(132 \%$; SEM, 26$)$ visual field $(p=0.73)$. Consistent with the results from the main experiment, speed thresholds for across-hemifield tracking were significantly higher relative to within-hemifield tracking $(p=0.005)$. Given these data, we are confident that the effects described here are due to the hemifield effect, and are not driven by other asymmetries (e.g., lower visual field advantage; He et al., 1996).

The individual threshold speeds were used in the EEG experiment to match performance across conditions. Accuracy with these adjusted speeds was fairly uniform, varying within a range of $80-88 \%$. An ANOVA was used to check how well performance was matched across conditions (one-target fast, onetarget slow, two-targets across, two-targets within); it did show a main effect of condition $\left(F_{(3,33)}=4.62, p=0.021, \eta^{2}=0.29\right)$, but importantly, there was no reliable difference between acrosshemifield relative to within-hemifield tracking $\left(p=0.176, \eta^{2}=\right.$ 0.11; Fig. 1C).
Early visual processing stages

Across all four conditions, the amplitude spectrum obtained by Fourier transformation shows clear peaks at the stimulating frequencies of the flickering target and distractor stimuli (Fig. 2). Targets elicited larger SSVEP amplitudes relative to the corresponding distractors. However, as can be seen in Figure $3 A$, the magnitude of the attentional modulation (target minus distractor) differed between tracking conditions $\left(F_{(3,33)}=4.20, p=\right.$ $\left.0.013, \eta^{2}=0.28\right)$, with particularly low attentional modulation in the two-target, within-hemifield condition. Follow-up contrasts confirmed that the attentional modulation during withinhemifield tracking was smaller compared with all other tracking conditions (Fig. $3 A$; within-hemifield vs across-hemifields: $t_{(11)}$ $=2.50, p=0.021, \eta^{2}=0.36$; within-hemifield vs one-target slow: $t_{(11)}=3.32, p=0.007, \eta^{2}=0.50$; within-hemifield vs one-target fast: $\left.t_{(11)}=2.85, p=0.006, \eta^{2}=0.47\right)$. Indeed, the SSVEP amplitude of targets did not differ reliably from distractors in the within-hemifield condition $\left(p=0.771, \eta^{2}=0.01\right)$, indicating that attention failed to effectively enhance visual processing for the two targets when they were presented in the same hemifield. In the across-hemifield tracking condition, the magnitude of the attentional modulation was smaller relative to single-target tracking at slow speed $\left(t_{(11)}=2.25, p=0.046, \eta^{2}=\right.$ $0.32)$, but did not differ from single-target tracking at the matched speed (i.e., fast; $p=0.380, \eta^{2}=0.07$ ).

In the second analysis, we compared attentional modulation of each target-distractor pair separately when participants tracked one or two targets at the same speed. As shown in Figure 
A Normalized attention effects (SSVEP),
collapsed across target-distractor pair

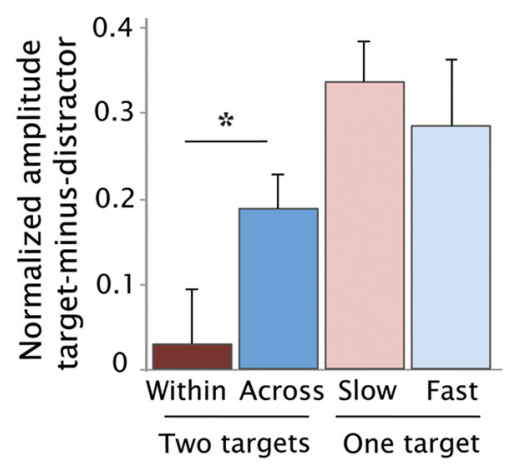

B

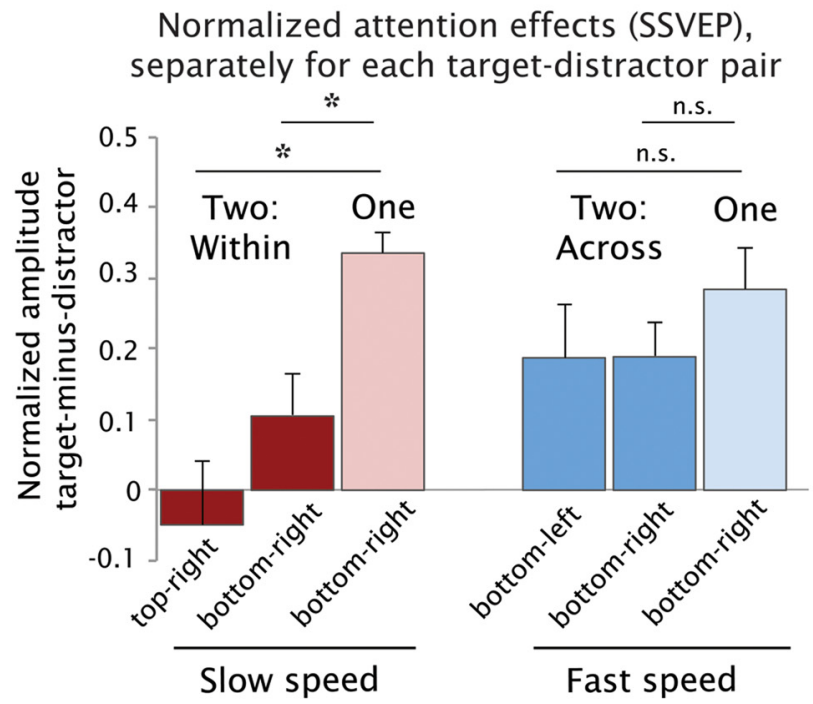

Figure 3. Normalized attentional modulation (target minus distractor) of early visual processing indexed by SSVEPS. A, The attentional modulation is lower when participants tracked two targets within a hemifield relative to two targets across hemifields, or one target at slow and fast speed. $\boldsymbol{B}$, Normalized attentional modulation for each target-distractor pair, separately for when the targets moved at slower speeds (and attention was divided within a hemifield, left side), and for when targets moved at faster speeds (and attention was divided across hemifields, right side). The magnitude of attentional modulation is reduced for both targets for within-hemifield tracking, but not for across-hemifield tracking relative to when tracking one target at the same speed. Error bars represent the SEM.

$3 B$, the magnitude of attentional modulation (target minus distractor) was significantly reduced for both target-distractor pairs when participants tracked two targets within the same hemifield, relative to when they only tracked one target at the same speed $\left(F_{(2,22)}=9.16, p=0.005, \eta^{2}=0.45\right)$. Pairwise comparisons confirmed that the attention effect was reduced for the targetdistractor pair in the top-right quadrant $\left(t_{(11)}=2.95, p=0.013\right.$, $\left.\eta^{2}=0.44\right)$, and also for the bottom-right quadrant $\left(t_{(11)}=2.42\right.$, $\left.p=0.034, \eta^{2}=0.34\right)$, relative to when participants tracked a single target in the bottom-right quadrant (Fig. $3 B$, left). In contrast, there were no reliable differences in the magnitude of the attention effects for either target-distractor pair when participants tracked two targets that appeared in separate hemifields (at fast speed) compared with when they tracked one target at the same speed (bottom-left vs single target: $t_{(11)}=0.695, p=0.502$, $\eta^{2}=0.04$; bottom-right vs single target: $t_{(11)}=0.908, p=0.384$, $\left.\eta^{2}=0.06\right)$. Note that sensory input was matched when comparing the SSVEP amplitudes elicited by the target-distractor pair in the bottom-right quadrant. Thus, any differences in SSVEP amplitudes must be attributed to differences in attentional processes.

\section{Later processing stages}

Test probes on targets elicited a larger P3 component than test probes on distractors across all conditions $\left(F_{(1,11)}=10.76, p=\right.$ $\left.0.03, \eta^{2}=0.16\right)$, showing that targets were effectively discriminated from distractors by later processing stages (Fig. 4). No effect of tracking condition and no interactions were observed, indicating that any across-hemifield advantage was not evident at these later processing stages, as might be expected given the similar values of tracking accuracy imposed across conditions.

\section{Discussion}

It is easier to select multiple targets when they appear in separate visual fields, compared with when targets appear in the same visual hemifield (Awh and Pashler, 2000; Alvarez and Cavanagh, 2005; Alvarez et al., 2012). In the present study we measured early attentional selection of multiple targets by tagging moving targets and distractors with distinct frequencies, and examined the SSVEP amplitudes elicited by these stimuli. When observers tracked two targets in separate visual fields (i.e., bottom-left and bottom-right), SSVEP amplitudes for each target were larger compared with the corresponding distractor, and not different from when tracking a single target at the same speed. In contrast, the SSVEP correlates of visual processing for targets and distractors were indistinguishable when observers tracked two targets in the same hemifield (i.e., bottom-right and top-right). These results are consistent with the possibility that the across-hemifield advantage arises at an early sensory processing level; namely, during stimulus processing itself. These results could not be explained by differences in task accuracy, which was controlled by manipulating target speed, or by differences in target speed, which alone had no effect on SSVEP modulation.

Previous studies have shown that attention can facilitate inputs from multiple separate locations at the level of the extrastriate occipital pathways (Müller et al., 2003; Störmer et al., 2013a). Here, we demonstrate that these early facilitatory effects of sustained multifocal attention are influenced by hemifield boundaries. A recent fMRI study showed two distinct peaks of retinotopically specific activation in primary visual cortex when participants divided attention across separate visual fields and within the same hemifield, suggestive of two distinct attentional "spotlights." When participants divided attention within a hemifield, however, there was also some attentional modulation in the intervening region, suggesting that attention was divided less effectively within a hemifield (McMains and Somers, 2004). The present study shows a much more dramatic decrease in attentional modulation for targets within the same hemifield, with no measurable difference between targets and distractors, even when task performance is adjusted so that two targets could be tracked successfully. This implies that early target enhancement may not be obligatory for selecting and tracking multiple targets, but appears to make it more effective (Störmer et al., 2013a).

Another recent paper showed attenuated target responses indexed by SSVEPs for stimuli appearing within the same visual hemifield relative to stimuli appearing in separate hemifields (Walter et al., 2014). In that study, participants were asked to 


\section{A}
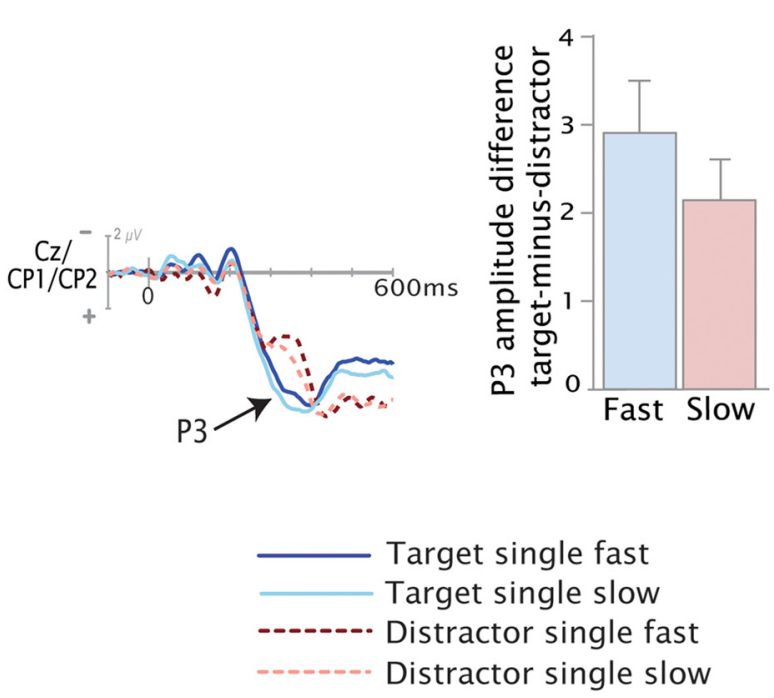

B

\section{Two targets tracked}
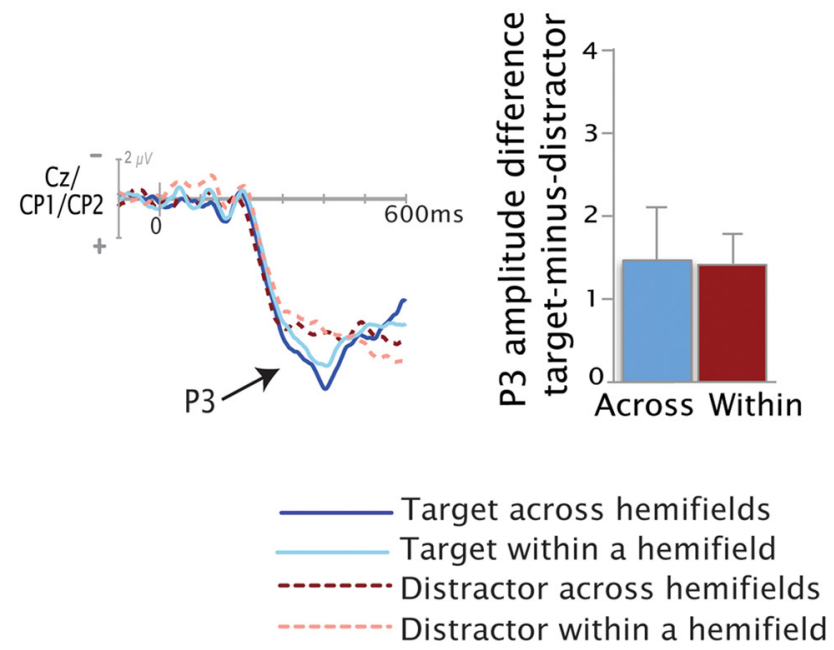

Figure 4. ERPs elicited by test probes on targets (blue, solid lines) and distractors (red, dashed lines) at central-parietal electrode sites. The P3 component was larger for targets relative to distractors across all conditions. A, ERPs elicited by probes on targets and distractors when participants tracked a single target only, either at fast (dark shading) or slow (light shading) speed. The bar graph depicts the target-minus-distractor mean amplitude differences of the $\mathrm{P3}$ component for tracking one target at fast and slow speed. $\boldsymbol{B}$, ERPs elicited by probes on targets and distractors when participants tracked two targets, either across visual hemifields (dark shading) or within a hemifield (light shading). The bar graph depicts the target-minusdistractor mean amplitude differences of the P3 component for across (fast speed) and within (slow speed) hemifield tracking. Error bars represent the SEM.

detect luminance increments of flashing light-emitting diodes in the same or in opposite visual hemifields. Detection performance was slightly better across hemifields than within a hemifield, although these behavioral differences (9.5\% across-hemifield advantage) were much smaller than the effect in our tracking task (230\% across-hemifield advantage), consistent with theories suggesting that hemifield effects arise primarily when filtering targets from among same-hemifield distractors using spatial attention (Alvarez et al., 2012; Franconeri et al., 2013). Correspondingly, the SSVEP responses in their study show a hemifield effect of smaller magnitude (133-300\%) than that found in the present study (523\%). Importantly, in Walter et al. (2014), this hemifield difference reflects target processing alone, and not the differential processing of targets relative to same-hemifield distractors; thus, the difference in SSVEP amplitudes could reflect a general increase in visual processing across hemifields, or, since behavior was not matched across the conditions, could be related to the lower task performance on within-hemifield trials.

\section{Early versus late selection mechanisms}

It seems surprising that early visual processing of targets and distractors was indistinguishable in the SSVEP responses during within-hemifield tracking, given that observers performed at $80 \%$ accuracy. However, it is important to note that, to achieve $80 \%$ accuracy, targets had to move more slowly in the within-hemifield condition than in the across-hemifield condition. One integrative interpretation of these results is that later processing stages can support tracking multiple targets, but that they do so less effectively (and thus can succeed only at slower speeds). This interpretation is consistent with the additional finding that, even for within-hemifield tracking of two targets, the targets were just as reliably discriminated from distractors as for across-hemifields tracking at a later processing stage, as reflected in amplitude modulations of the P3 component, which was measured over central-parietal cortex. This implies that the differences in target selection at earlier stages, as indexed by the SSVEPs, were successfully compensated for at other higher processing levels. However, this only seems to be the case when targets move at slow speeds, but not when targets move at fast speeds. This suggests that higherlevel processes can support multiple-target tracking, but that the rate at which they can update representations is slower, preventing them from consistently tracking multiple fastmoving targets.

\section{Hemifield independence}

Consistent with previous research (Alvarez and Cavanagh, 2005), we found evidence for independent selection of targets in the left and right visual fields. We also showed through direct electrophysiological measures that this independence is reflected in modulations of early visual areas: target modulations measured by SSVEP were just as strong for a single target as for multiple targets across hemifields. Overall, the pattern of data diverges from models that assume a centrally organized attention process that distributes a unified resource equally across targets in the visual field (Broadbent, 1958; Moran and Desimone, 1985). Instead, it supports a model in which early target processing is facilitated independently for the left and right visual fields.

What mechanisms give rise to hemifield independence in early visual processing? The attentional modulations observed in early sensory processing pathways are likely governed by topdown bias signals from parietal and frontal brain regions. Previous fMRI studies have found load-dependent activation in parietal brain regions during multiple-object tracking (Culham et al., 2001; Jovicich et al., 2001; Howe et al., 2009). This suggests that activity in parietal brain areas reflects control mechanisms that appoint attentional resources to targets during tracking.

This framework suggests two broad categories of explanation for hemifield independence, one relating to competition between representations in early visual maps, and the other related to lateralized control mechanisms. For example, while spatial atten- 
tion enhances target processing, it simultaneously suppresses processing of surrounding irrelevant information (Mounts, 2000; Cutzu and Tsotsos, 2003; Müller and Kleinschmidt, 2004; Müller et al., 2005; Hopf et al., 2006). If these suppressive mechanisms relied on lateral connections that are stronger within a hemifield than across hemifields because of separate hemifield representations in lower-level visual areas (V1, V2, V3, lateral occipital cortex, and middle temporal cortex; Bullier, 2004; Gardner et al., 2008), then suppression zones would overlap more within the same visual field, leading to greater suppression between targets within a hemifield, and thus more confusion of targets and distractors within a hemifield (Alvarez et al., 2012; Franconeri, 2013; Franconeri et al., 2013), and less distinct SSVEP responses for targets and distractors within a hemifield.

Alternatively, it is possible that there exist independent attentional pointers for each hemifield. When targets are presented in separate visual fields, these pointers can reliably allocate resources to each target in parallel, thereby facilitating their processing effectively_-just as effectively as for a single target. When targets are presented in the same visual hemifield, however, only one pointer is available. This pointer would need to switch back and forth between the targets in a serial manner, leading to less effective modulation of early visual areas. This possibility is consistent with results from transcranial magnetic stimulation (TMS) studies, which show that TMS applied to the parietal lobes impairs tracking in the contralateral hemifield (when multiple objects are tracked; Battelli et al., 2009).

Both hypotheses are consistent with our finding of reduced attentional facilitation of targets when they appear in the same visual hemifield, and further research will be necessary to determine the extent to which map-based competition versus lateralized attentional control account for hemifield independence.

\section{Conclusion}

Our results show that the benefits of dividing attention across separate visual hemifields emerge at an early sensory level, namely during stimulus processing itself. Visual processing of multiple targets as indexed by SSVEP signals is continuously facilitated when attention is divided across the left and right visual field, possibly as effectively as for a single target. However, these early facilitatory effects are greatly attenuated when attention is divided within the same visual hemifield. Higher-level identification and discrimination processes, on the other hand, do not seem to be affected by how attention is divided in the visual field, but operate less effectively, possibly reflecting the slow clock-rate of central processing. This leads us to propose that when attention is divided within a hemifield, higher-level attentional pointers fail to reliably reach down to early visual areas to facilitate sensory processing of multiple-target stimuli. Overall then, our results point to dramatic constraints of attentional selection beginning at an early sensory processing stage. They indicate that the mechanisms of attentional selection are limited by the architecture of the visual system, and that the capacity of the system to process multiple items at once is determined by the combination of constraints at multiple levels of processing.

\section{References}

Alvarez GA, Cavanagh P (2005) Independent resources for attentional tracking in the left and right visual hemifields. Psychol Sci 16:637-643. CrossRef Medline

Alvarez GA, Gill J, Cavanagh P (2012) Anatomical constraints on attention: hemifield independence is a signature of multifocal spatial selection. J Vis 12(5):9. CrossRef Medline

Andersen SK, Müller MM, Hillyard SA (2011a) Tracking the allocation of attention in visual scenes with steady-state evoked potentials. In: Cognitive neuroscience of attention, second edition (MI Posner, ed). New York: Guilford.

Andersen SK, Fuchs S, Müller MM (2011b) Effects of feature-selective and spatial attention at different stages of visual processing. J Cogn Neurosci 23:238-246. CrossRef Medline

Awh E, Pashler H (2000) Evidence for split attentional foci. J Exp Psychol Hum Percept Perform 26:834-846. Medline

Battelli L, Alvarez GA, Carlson T, Pascual-Leone A (2009) The role of the parietal lobe in visual extinction studied with transcranial magnetic stimulation. J Cogn Neurosci 21:1946-1955. CrossRef Medline

Broadbent DE (1958) Perception and communication. London: Pergamon.

Bullier J (2004) Communications between cortical areas of the visual system. In: The visual neurosciences (Chalupa LM, Werner JS, eds), pp 522 540. Cambridge, MA: MIT.

Chakravarthi R, Cavanagh P (2009) Bilateral field advantage in visual crowding. Vis Res 49:1638-1646. CrossRef Medline

Culham JC, Cavanagh P, Kanwisher NG (2001) Attention response functions: characterizing brain areas using $\mathrm{fMRI}$ activation during parametric variations of attentional load. Neuron 32:737-745. CrossRef Medline

Cutzu F, Tsotsos JK (2003) The selective tuning model of attention: psychophysical evidence for a suppressive annulus around an attended item. Vis Res 43:205-219. CrossRef Medline

Eriksen CW, Yeh YY (1985) Allocation of attention in the visual field. J Exp Psychol Hum Percept Perform 11:583-597. CrossRef Medline

Franconeri SL (2013) The nature and status of visual resources. In: Oxford handbook of cognitive psychology (Reisberg D, ed). New York: Oxford UP.

Franconeri SL, Alvarez GA, Cavanagh P (2013) Flexible cognitive resources: competitive content maps for attention and memory. Trends Cogn Sci 17:134-141. CrossRef Medline

Gardner JL, Merriam EP, Movshon JA, Heeger DJ (2008) Maps of visual space in human occipital cortex are retinotopic, not spatiotopic. J Neurosci 28:3988-3999. CrossRef Medline

Green JJ, Conder JA, McDonald JJ (2008) Lateralized frontal activity elicited by attention-directing visual and auditory cues. Psychophysiology 45: 579-587. CrossRef Medline

He S, Cavanagh P, Intriligator J (1996) Attentional resolution and the locus of visual awareness. Nature 383:334-337. CrossRef Medline

Hillyard SA, Picton TW (1987) Electrophysiology of cognition. In: Handbook of physiology (Plum F, ed), pp 519-584. Bethesda, MD: American Physiological Society.

Holcombe AO, Chen WY (2012) Exhausting attentional tracking resources with a single fast-moving object. Cognition 123:218-228. CrossRef Medline

Hopf JM, Boehler CN, Luck SJ, Tsotsos JK, Heinze HJ, Schoenfeld MA (2006) Direct neurophysiological evidence for spatial suppression surrounding the focus of attention in vision. Proc Natl Acad Sci U S A 103: 1053-1058. CrossRef Medline

Howe PD, Horowitz TS, Morocz IA, Wolfe J, Livingstone MS (2009) Using fMRI to distinguish components of the multiple object tracking task. J Vis 9(4):1-11. CrossRef Medline

Hudson C, Howe PD, Little DR (2012) Hemifield effects in multiple identity tracking. PloS One 7:e43796. CrossRef Medline

Jovicich J, Peters RJ, Koch C, Braun J, Chang L, Ernst T (2001) Brain areas specific for attentional load in a motion-tracking task. J Cogn Neurosci 13:1048-1058. CrossRef Medline

LaBerge D (1995) Attentional processing: the brain's art of mindfulness. Cambridge, MA: Harvard UP.

McMains SA, Somers DC (2004) Multiple spotlights of attentional selection in human visual cortex. Neuron 42:677-686. CrossRef Medline

Moran J, Desimone R (1985) Selective attention gates visual processing in the extrastriate cortex. Science 229:782-784. CrossRef Medline

Morgan ST, Hansen JC, Hillyard SA (1996) Selective attention to stimulus location modulates the steady-state visual evoked potential. Proc Natl Acad Sci U S A 93:4770-4774. CrossRef Medline

Mounts JR (2000) Evidence for suppressive mechanisms in attentional selection: feature singletons produce inhibitory surrounds. Percept Psychophys 62:969-983. CrossRef Medline

Müller MM, Malinowski P, Gruber T, Hillyard SA (2003) Sustained division of the attentional spotlight. Nature 424:309-312. CrossRef Medline 
Müller NG, Kleinschmidt A (2004) The attentional "spotlight's" penumbra: center-surround modulation in striate cortex. Neuroreport 15:977980. CrossRef Medline

Müller NG, Mollenhauer M, Rösler A, Kleinschmidt A (2005) The attentional field has a Mexican hat distribution. Vis Res 45:1129-1137. CrossRef Medline

Polich J (2007) Updating P300: an integrative theory of P3a and P3b. Clin Neurophysiol 118:2128-2148. CrossRef Medline

Reardon KM, Kelly JG, Matthews N (2009) Bilateral attentional advantage on elementary visual tasks. Vis Res 49:691-701. CrossRef Medline

Regan D (1989) Human brain electrophysiology: evoked potentials and evoked magnetic fields in science and medicine. New York: Elsevier.

Sereno AB, Kosslyn SM (1991) Discrimination within and between hemifields: a new constraint on theories of attention. Neuropsychologia 29: 659-675. CrossRef Medline

Squires KC, Hillyard SA, Lindsay PH (1973) Vertex potentials evoked dur- ing auditory signal detection: relation to decision criteria. Percept Psychophys 14:265-272. CrossRef

Störmer VS, Winther GN, Li SC, Andersen SK (2013a) Sustained multifocal attentional enhancment of stimulus processing in early visual areas predicts tracking performance. J Neurosci 33:5346-5351. CrossRef Medline

Störmer VS, Li SC, Heekeren HR, Lindenberger U (2013b) Normal aging delays and compromises early visual attentional selection: evidence from event-related potentials during multiple-object tracking. J Cogn Neurosci 25:188-202. CrossRef Medline

Walter S, Quigley C, Mueller MM (2014) Competitive interactions of attentional resources in early visual cortex within or between visual hemifields during sustained visuospatial attention: evidence for the differenthemifield advantage. J Cogn Neurosci 26:938-954. CrossRef Medline

Watson AB, Pelli DG (1983) QUEST: a Bayesian adaptive psychometric method. Percept Psychophys 33:113-120. CrossRef Medline 